\title{
Eigenface Based Recognition of Emotion Variant Faces
}

\author{
${ }^{1}$ Thuseethan, S., ${ }^{2}$ Kuhanesan, S. \\ ${ }^{1}$ Department of Computing and Information Systems, Sabaragamuwa University of Sri Lanka, Sri Lanka \\ ${ }^{2}$ Department of Physical Science, Vavuniya Campus of the University of Jaffna, Sri Lanka \\ Email of corresponding author thuseethan@gmail.com
}

\begin{abstract}
In present, the automatic machine based face recognition has become significant due to its urgency in potential application and current scientific challenges of industries. However, most of the existing systems designed up to now can only effectively distinguish the faces when source images are collected under numerous constrained conditions. The success rate or positive impact of face recognition systems depend on a variety of information imposed in images of human faces. Pose of face, facial expression, angle, occlusion and state of structural components are some of those. Emotions can be expressed in different ways that can be seen such as facial expression, speech, written text and gestures. This model propose an efficient approach for the recognition of expression or emotion variant faces since there are very few emotion recognition software tools to handle such problems and there is a significant importance to this research area in the field of face recognition. Especially an approach proposed here to face recognition where the facial expression in the training image set and in the testing image set diverge and only one sample image per class is existing in the system. The input to the system is a frontal neutral expression oriented face image with unique background. In this image the hair is tied away from the face and facial hair should be removed. Principal Component Analysis approach was used as a primary mechanism in the proposed model. This approach has been applied purely on a set of face images in order to extract a set of eigenface images as the output. Here weights of the representation or image are used for recognition of emotions. One of the distance metric approaches Euclidean Distance used to discover the distance with the weight vectors which was associated with each of the training images for the existence of classification task.
\end{abstract}

Keywords: Face Recognition, Emotion-variant faces, Image Processing, Principal Component Analysis, Euclidean Distance

\section{Introduction}

As one of the most successful applications of image analysis and understanding, face recognition has recently received significant attention, especially during the past several years [Zhao, 2003]. Face has gained significant importance in identifying the state and expressions of a human. Race, sex, age and emotions are some major information can be extracted or identified by analyzing human faces. Human faces are complex set of objects with so many features that can differ all the time based on influence of external environment. Due to technological advancements; there is an arousal of the world where human being and intelligent robots live together [Khandait et al., 2011]. Recognizing the emotional state of a person by analyzing him/her facial expression appear challenging but also essential many times due to the fact that most of the times facial expressions are not only the direct emotional outcome but also result from the extraction of the meaning of concepts. However, humans have the natural and inbuilt ability to detect, recognize, differentiate and identify or pinpoint a person's face. Computer is a machine which made by man and now he is trying to give artificial brain to go hand in hand with the human mind [Thuseethan, 2014]. On the other hand unfortunately machines do not have this kind of capability, but can be imposed through learning.

The human sciences comprise a bank of literature based on emotion which is huge, but can be fragmented. Expression and feeling of people differs under different situations with different context. Recognizing the emotion of the face plays a significant role in the human-computer interaction. One of the key challenges for face recognition is finding efficient and discriminative facial appearance descriptors that are resistant to large variations in illumination, facial expression, pose, ageing, partial occlusions and other changes [Tan and Triggs, 2007]. The success of face recognition systems depend on a variety of information included in images of human faces such as pose, facial expression, occlusion, condition of image and presence or absence of structural components [Khashman, 2008]. Current systems have advanced to be fairly accurate in recognition under constrained scenarios, but extrinsic imaging parameters such as pose, illumination, and facial expression still cause much difficulty in correct recognition [Huang et al.,2002]. The important sources which are significant to this approach are in human psychology and linguistics, with some shadow from biology. A less studied problem 
is the recognition of expression variant faces; this problem can be formulated as follows: how can we robustly identify a person's face for which the learning and testing images differ in facial expressions? [Martinez, 2003].

The importance of recognizing emotion or expression variant faces is its ability to recognize criminals, suspects and employees of an organization. Even with one and only image it can recognize the emotion. The main objective of this research is to develop an Automatic Facial Expression Recognition System (AFERS) which automatically gathers human facial images containing some expression as input and recognize and classify it into appropriate expression class such as happy, angry, fear, disgust, neutral, surprise, and sad. One person may have too many different expressions or emotions at different times [Piquepaille, 2008]. Finally it identifies the image which has same person's face with different expressions or emotions. For example in a security division the police officer able to match the face image extracted from video or security cameras with available face images stored in system, for the purpose of identify a criminal or suspect. As mentioned previously, few drawbacks exist in newly developed system. But the proposed approach overcomes some of those drawbacks completely such as supports for single training face image.

\section{Proposed Model}

The proposed system comprises of four major modules. Those are Face Library, Pre-processing, Generate eigenfaces and Process Recognition. Figure 1 shows the block diagram of interrelation and flow of process between four major modules.

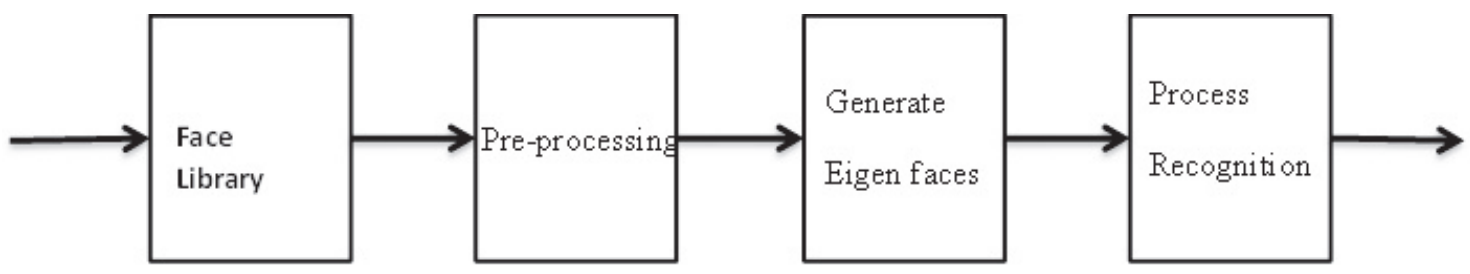

Figure 1: Block diagram for flow of process

\subsection{Face Library}

In the training phase user should be able to add images to face library, the user can load the image from any area of disk and he/she will give personal details. These images will be saved into a specific folder and personal details regarding the image will be saved in a database developed in Microsoft Access. Here a real time database used instead of download the images from an existing database. The reason behind this is because real time databases noise free and easy use. The loaded training images will be converted into a vector format data and will be saved in MATLAB by using an ASCII file with the ".dat" extension. This generated data file will be moderated with two columns to store one image vector and a class number. Here the class number represents the unique number for each training image belongs to a particular person.

\subsection{Pre-processing}

The frontal face images with same background are the acceptable input format for the system. Further the image should be converted in such a way that the system understands or supports. The systems detect face under controlled conditions, such as without glasses, facial hair and any rigid head movement. Therefore, the input image should satisfy the above conditions. First the image has to be converted to grayscale by get rid of the hue (color) and diffusion information while retaining the luminance. All images are represented in matrix form and to gain maximum efficiency in matrix operations all of them attained as square matrix with the same size and are homogenized. The image inputs by user may vary in practice, and it is not a good restrict the user to use images in a pre-given size all the time. This system let the user to input image with any size and handle the resize mechanism internally after the insertion of image. System resizes all images to $480 * 480$ pixel scales for further processing.

\subsection{Generate Eigenfaces}

The author has identified eigenface approach as an appropriate method to recognize expression variant faces after conducting a broad literature review because it has advantages over other face recognition methods such as speed, simplicity and learning capability [Turk \& Pentland, 1991]. The Eigen face method is based on linearly projecting the image space to a low dimensional feature space [Peter N. Belhumeur et al., 1997]. Before eigenfaces were introduced in face recognition literature, most of the face recognition researches dealt with local 
features such as distance between ears, eyes and similar other features, this was not effective. eigenfaces is a Principal Component Analysis (PCA) approach, which uses a small set of characteristic pictures to describe the variations between face images. These eigenfaces will be derived from the covariance matrix of the distribution of high dimensional vector space of training set of face images. However, the identified eigenface method, which uses principal components analysis (PCA) for dimensionality reduction, yields projection directions that maximize the total scatter across all classes, i.e., across all images of all faces [Belhumeur et al., 1997]. Here, the original face image of training set will be transformed into a corresponding Eigen face and every face image is represented by a linear combination of these eigenvectors. The steps to find eigenfaces are:

Step 1: Call the vector of all the training images saved in the ASCII file and stores in a matrix.

$$
\mathrm{T}=\left\{\mathrm{T}_{1}, \mathrm{~T}_{2}, \mathrm{~T}_{3} \ldots . . \mathrm{T}_{\mathrm{N}}\right\}
$$

The above equation indicates a set $\mathrm{T}$ which comprises all the training images and $\mathrm{N}$ is number of available or existing training images stored in database.

Step2: Find the mean of loaded training images using the equation given below.

$$
\Psi=\frac{1}{N} \sum_{m=1}^{N} \prod_{m}
$$

$\Psi=$ Mean of the training set

$\Gamma m=$ Vector of Training image

$\mathrm{N}=$ Number of training images

Step3: Subtract the calculated mean from the vector of training image to find subtracted mean

$$
\Phi_{m}=\Gamma_{m}-\mathbf{Y}
$$

$\Phi_{m}=$ The subtracted vector of training image

$\Gamma m=$ Vector of training image

$\Psi=$ Mean of the training set

Here, the $\Phi_{m}$ indicates the difference between the vector of $\mathrm{m}^{\text {'th }}$ training image and the mean image.

Step4: Calculate the covariance matrix for the subtracted vector of training image.

$$
C=\frac{1}{N} \sum_{m=1}^{N} \boldsymbol{\Phi}_{m} \boldsymbol{\Phi}_{m}^{T}
$$

Here, $\mathrm{C}$ is the covariance matrix of matrix which has subtracted image vectors; it can be simply specified as like this,

Step 5: Calculate Eigen vectors and Eigen values, eigenvectors and corresponding eigenvalues should be calculated from covariance matrix C.

$$
\mathrm{C}=\mathrm{AAT}
$$

$$
A=\left[\Phi_{1}, \Phi_{2}, \Phi_{3} \ldots . . \Phi_{N}\right]
$$

Here, $\mathrm{C}$ is a V2xV2 matrix and $\mathrm{A}$ is a V2 $\mathrm{x} \mathrm{N}$ matrix

Step6: Obtaining the appropriate eigenfaces

In this step, the Eigenvectors that have highest Eigen values will be chose as the well fit eigenface because it describes more characteristic features of the particular selected face. Eigenfaces with low Eigen values could be omitted since they matched only a small part of characteristic features of the faces. 


\subsection{Process Recognition}

Find the weight vector of the stored images: To initialize the recognition task, at first the weight vector of the training images should be derived and calculated. And these vectors have to be stored corresponding to respective training image.

Load testing image: Then the testing image will be loaded by the user and the system will convert this into a vector. Before do this some pre-processing operations should be handled and done to the testing image in order to improve the quality of the image to support to do better recognition of face.

Find feature vector: After loaded the training image, the feature vector of the training image can be extracted and found. Here the new facial image is transformed into its eigenface components by a well-defined simple operation.

Calculate Euclidean Distance: Classification will be performed by comparing the feature vectors of the training images with feature vector of input testing image. Distance measure is an important part of a vector model [Qian, 2004]. It can be done by using the distance measures; Euclidean Distance is probably the most widely used distance metric. The following algorithms illustrates this clearly,

$$
d_{i}=\min \left\|\Omega-\Omega_{i}\right\|
$$

$d_{i}=$ Distance between the testing image and training image $\mathrm{i}$

$\Omega=$ Feature vector of particular testing image

$\Omega_{i}=$ Weight vector of the training image $\mathrm{i}$

Here, the weight vector of the testing image has been considered to take and find its distance with the weight vectors associated with each of the training image.

Choose Threshold: An optimal threshold is selected by the discriminant criterion, namely, so as to maximize the separability of the resultant classes in gray levels [Otsu, 1975]. The threshold used in a scenario where the face belongs to a testing image does not have a training image exist in database. The distance for each training image will be found out with the input testing image with respect to given image information. And even if the training image for a person does not exist in the database; still it will say the person is recognized as the training image with which scores is the lowest. Clearly this indicates an obstacle, to overcome this issue the threshold must be decided as $\Theta$. A threshold should be chosen that defines the maximum allowable distance from any face image. If $\mathrm{di}<\Theta$, then the testing image is recognized as the image with which it gives the lowest distance. If $d_{i}>\Theta$, then the person belonging to that testing image does not belong to the database.

Display Results: If the comparison satisfies the threshold or find suitable image for at least one member then the face image will be classified as "Known face", otherwise it will be classified as "Unknown face". Once the face image is classified as "Known face" then the personal details are those saved in database will be displayed for further clarification through interface. Well organized interfaces used here to represent the result; it will increase the usability of the system [Thuseethan and Kuhanesan, 2014]. If it is classified as "unknown face" then the message "Match is not found" will be displayed.

\section{Testing and Discussion}

Publically available face data set JAFFE (Japanese Female Facial Expressions) used in order to test the prototype. JAFFE database is commonly used in measuring the performance of facial expression recognition systems [Shih et al., 2008]. Although other face databases with large number of subjects exist, they are inappropriate to test this prototype under some criteria such as they do not have enough emotion variant faces. JAFFE dataset is used as the standard database in performance comparisons of facial expression recognition in JAFFE Database researches [Buciu et al., 2003; Dubuisson et al., 2002; Lyons et al., 1999; Shinohara and Otsu, 2004; Yang et al., 2004].

This database contains ten Japanese females. In JAFFE dataset, eight participants posed of six of the basic expressions such as happiness, sadness, surprise, anger, disgust and fear. Neutral faces were also photographed. For each female there are up to four examples for each expression available. Figure 4 shows one such example with three different representation of sadness. There are 213 different gray scale facial expression images in this 
database. All images are of size $256 \times 256$ pixels. The experimental photos give frontal face views and hair was tied away from the face to make all areas of the face visible as required by the system. Light also was controlled. The following figure 2 shows the images of eight people with neutral expression used for training.
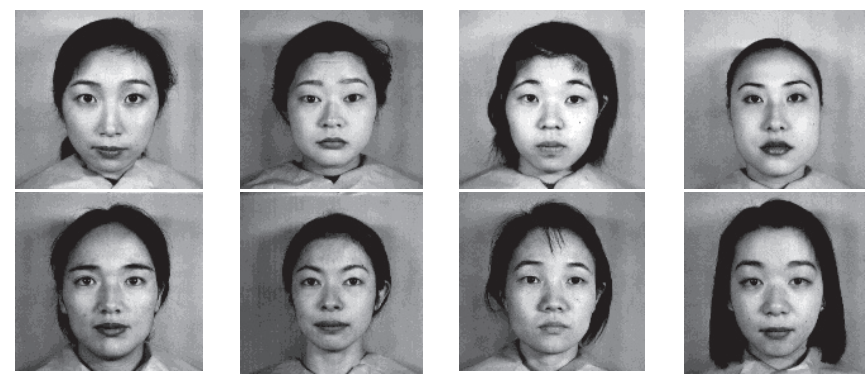

Figure 2: Images of sample females used in testing

The below Figure 3 shows six of the basic expressions such as fear, happy, angry, disgust, sadness and surprise of a random sample person. Further it shows the neutral face of that particular person.

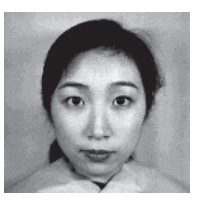

Neutral

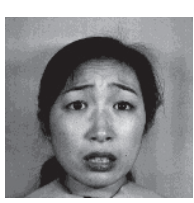

Fear

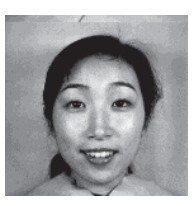

Happy

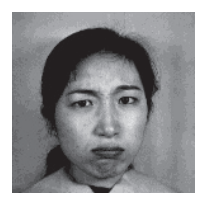

Angry

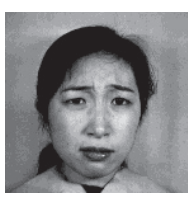

Disgust

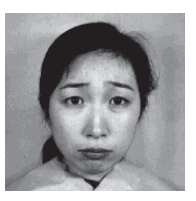

Sad

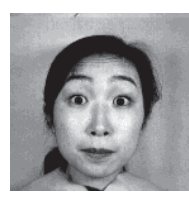

Surprise

Figure 3: Images with different emotions used for training from JAFFE database
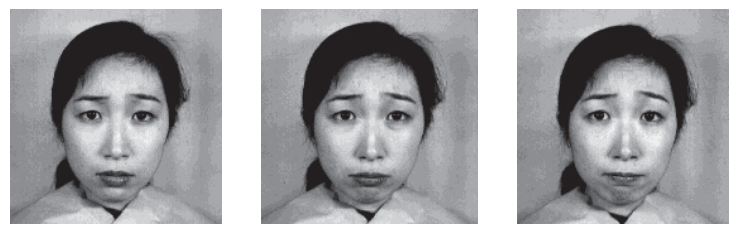

Figure 4: Different takes for single emotion (Sadness)

Table 1 show the recognitions results where the performance is evaluated according to the Cumulative Match Characteristic (CMC) metric defined by following equation [Grother and Micheals, 2003].

$$
P R(r)=\frac{|C(r)|}{|T|} .100
$$


Here, $\mathrm{C}(\mathrm{r})$ is testing image set recognized correctly in the set $\mathrm{T}$

\begin{tabular}{|c|c|}
\hline Test Set & Result \\
\hline $\operatorname{PR}(1)$ & $100 \%$ \\
\hline $\operatorname{PR}(2)$ & $92 \%$ \\
\hline $\operatorname{PR}(3)$ & $97 \%$ \\
\hline $\operatorname{PR}(4)$ & $93 \%$ \\
\hline $\operatorname{PR}(5)$ & $96 \%$ \\
\hline $\operatorname{PR}(6)$ & $100 \%$ \\
\hline $\operatorname{PR}(7)$ & $99 \%$ \\
\hline $\operatorname{PR}(8)$ & $100 \%$ \\
\hline
\end{tabular}

Table 1: Recognitions results of each subject

In this paper, we investigate the recognition of feature representation or expression variant faces schemes with seven different facial expressions on the JAFFE database. The overall efficiency of this system is good while having some deviations in recognition. The following table 2 shows the details of recognition rates of each and every expression with success percentage.

\begin{tabular}{|l|c|}
\hline Test Set & Result \\
\hline Anger & $100 \%$ \\
\hline Disgust & $85 \%$ \\
\hline Fear & $87 \%$ \\
\hline Happy & $100 \%$ \\
\hline Sad & $100 \%$ \\
\hline Surprise & $75 \%$ \\
\hline
\end{tabular}

Table 2: Recognitions results of each expression faces

\section{Conclusion}

\subsection{Limitations}

The accuracy is not sufficient while considering about try to recognize a person for whom there is no training image is stored in database.

This prototype currently has been tested with limited file types such as jpg, gif and tiff. Unable to test with other currently use types of image files such as bmp and png types.

Due to limitation and lack of available facial images the achieved outcomes may differ slightly with other image types.

The prototype has been tested with JAFFE facial image database; those databases have Chinese or Japanese faces and no other nationality faces. Due to this limitation in testing the system should be tested with other nationalities especially with some local faces.

\subsection{Enhancements}

Further real-time face recognition using a real time video input such as web cam can be made available to the user in order to reduce the human interaction with the system in the sense the implementation of suggested design improves the efficiency [Thuseethan and Kuhanesan, 2014].

Due to the existence of sunglasses, scarves, beards and mustaches there is a requirement to a mechanism to recognize real occluded face images.

Implementing multiple recognition feature embedded with the system such as voice, so that the user can get both visual and audio of the user verified result together, which would give ultimate secured login feature and trust on the system.

Implementing the system with the capabilities to recognize the face with dissimilar poses as well as detect the face in fair and complex and different backgrounds will make a complete system. 
Implement or expand the system in order to recognize different nationalities will produce an ideal system.

\section{References}

[1] Atalay, I. (1996). Face recognition using eigenfaces (Doctoral dissertation).

[2] Belhumeur, P. N., Hespanha, J. P., \& Kriegman, D. (1997). Eigenfaces vs. fisherfaces: Recognition using class specific linear projection. Pattern Analysis and Machine Intelligence, IEEE Transactions on, 19(7), 711-720.

[3] Buciu, I., Kotropoulos, C., \& Pitas, I. (2003, September). ICA and Gabor representation for facial expression recognition. In Image Processing, 2003. ICIP 2003. Proceedings. 2003 International Conference on (Vol. 2, pp. II-855). IEEE.

[4] Dubuisson, S., Davoine, F., \& Masson, M. (2002). A solution for facial expression representation and recognition. Signal Processing: Image Communication, 17(9), 657-673.

[5] Eleyan, A., \& Demirel, H. (2007). Pca and lda based neural networks for human face recognition. Face Recognition, 93-106.

[6] Fan, C., Sarrafzadeh, H., Dadgostar, F., Gholamhosseini, H., \& NSMC, A. (2005, November). Facial expression analysis by support vector regression. InProceedings of the Image and Vision Computing New Zealand Conference.

[7] Franc, V., \& Hlavác, V. (2004). Statistical pattern recognition toolbox for Matlab. Prague, Czech: Center for Machine Perception, Czech Technical University.

[8] Huang, J., Blanz, V., \& Heisele, B. (2002, August). Face recognition with support vector machines and 3D head models. In International Workshop on Pattern Recognition with Support Vector Machines (SVM2002) (pp. 334-341).

[9] Khashman, A. (2008). Intelligent local face recognition. Recent Advances in Face Recognition.

[10] Khandait, S. P., Thool, R. C., \& Khandait, P. D. (2012). Automatic facial feature extraction and expression recognition based on neural network. arXiv preprint arXiv:1204.2073.

[11] Kim, K. (1996). Face recognition using principle component analysis. InInternational Conference on Computer Vision and Pattern Recognition (pp. 586-591).

[12]Lyons, M., Akamatsu, S., Kamachi, M., \& Gyoba, J. (1998, April). Coding facial expressions with gabor wavelets. In Automatic Face and Gesture Recognition, 1998. Proceedings. Third IEEE International Conference on (pp. 200-205). IEEE.

[13]Lyons, M. J., Budynek, J., \& Akamatsu, S. (1999). Automatic classification of single facial images. IEEE Transactions on Pattern Analysis and Machine Intelligence, 21(12), 1357-1362.

[14] Martínez, A. M. (2003, June). Recognizing expression variant faces from a single sample image per class. In Computer Vision and Pattern Recognition, 2003. Proceedings. 2003 IEEE Computer Society Conference on (Vol. 1, pp. I-353). IEEE.

[15] Otsu, N. (1975). A threshold selection method from gray-level histograms.Automatica, 11(285-296), 23-27.

[16] Shih, F. Y., Chuang, C. F., \& Wang, P. S. (2008). Performance comparisons of facial expression recognition in JAFFE database. International Journal of Pattern Recognition and Artificial Intelligence, 22(03), 445-459.

[17] Shinohara, Y., \& Otsu, N. (2004, May). Facial expression recognition using fisher weight maps. In Automatic Face and Gesture Recognition, 2004. Proceedings. Sixth IEEE International Conference on (pp. 499-504). IEEE.

[18] Tan, X., \& Triggs, B. (2007). Fusing Gabor and LBP feature sets for kernel-based face recognition. In Analysis and Modeling of Faces and Gestures (pp. 235-249). Springer Berlin Heidelberg.

[19] Thuseethan, S. (2014). Department Management System For Departments Of Sri Lankan Universities. International Journal Of Scientific \& Technology Research, 3(6), 173-175.

[20] Thuseethan, T., \& Kuhanesan, S. (2014). Effective Use of Human Computer Interaction in Digital Academic Supportive Devices.International Journal of Science and Research, 3(6), 388-392.

[21] Turk, M., \& Pentland, A. (1991). Eigenfaces for recognition. Journal of cognitive neuroscience, 3(1), 71-86.

[22] Qian, G., Sural, S., Gu, Y., \& Pramanik, S. (2004, March). Similarity between Euclidean and cosine angle distance for nearest neighbor queries. InProceedings of the 2004 ACM symposium on Applied computing (pp. 1232-1237). ACM.

[23] Yang, J., Zhang, D., Frangi, A. F., \& Yang, J. Y. (2004). Two-dimensional PCA: a new approach to appearance-based face representation and recognition.Pattern Analysis and Machine Intelligence, IEEE Transactions on, 26(1), 131-137.

[24]Zhao, W., Chellappa, R., Phillips, P. J., \& Rosenfeld, A. (2003). Face recognition: A literature survey. Acm Computing Surveys (CSUR), 35(4), 399-458. 
The IISTE is a pioneer in the Open-Access hosting service and academic event management. The aim of the firm is Accelerating Global Knowledge Sharing.

More information about the firm can be found on the homepage:

http://www.iiste.org

\section{CALL FOR JOURNAL PAPERS}

There are more than 30 peer-reviewed academic journals hosted under the hosting platform.

Prospective authors of journals can find the submission instruction on the following page: http://www.iiste.org/journals/ All the journals articles are available online to the readers all over the world without financial, legal, or technical barriers other than those inseparable from gaining access to the internet itself. Paper version of the journals is also available upon request of readers and authors.

\section{MORE RESOURCES}

Book publication information: http://www.iiste.org/book/

\section{IISTE Knowledge Sharing Partners}

EBSCO, Index Copernicus, Ulrich's Periodicals Directory, JournalTOCS, PKP Open Archives Harvester, Bielefeld Academic Search Engine, Elektronische Zeitschriftenbibliothek EZB, Open J-Gate, OCLC WorldCat, Universe Digtial Library, NewJour, Google Scholar

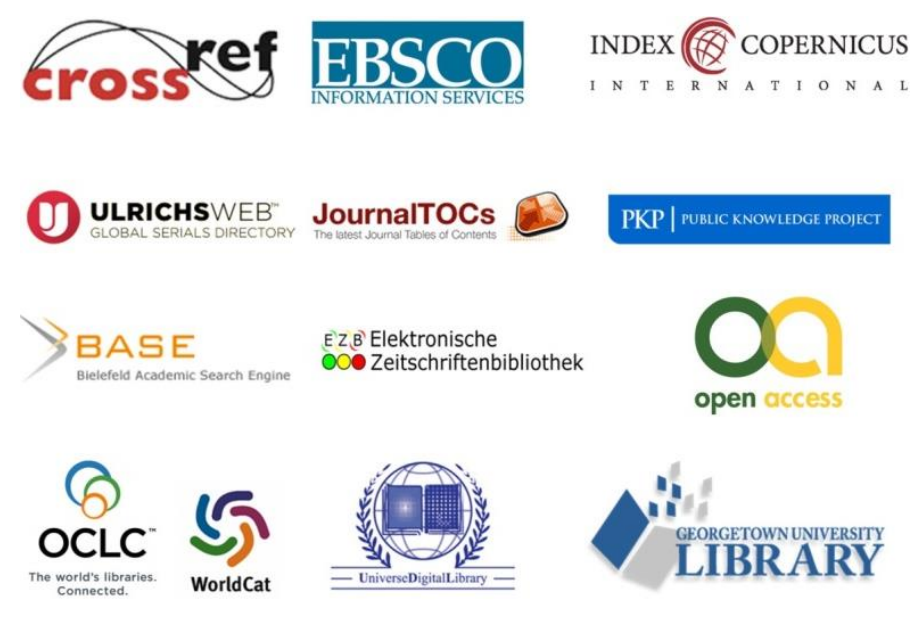

